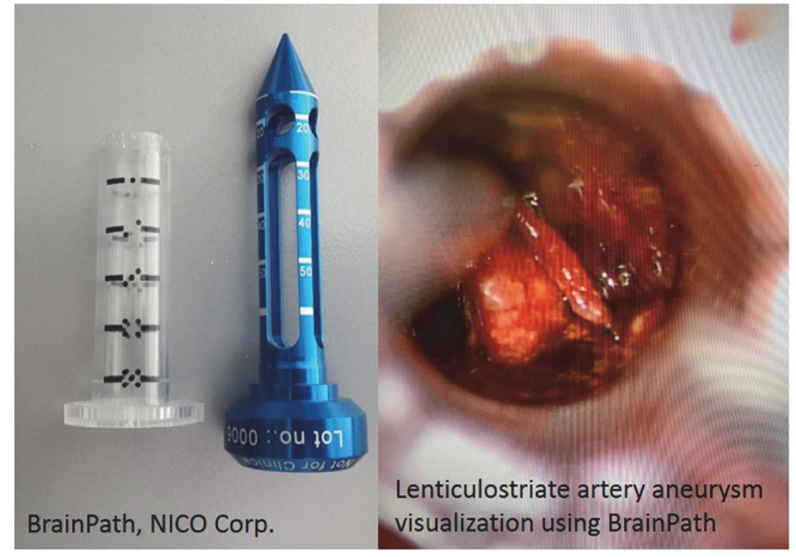

Abstract E-096 Figure 1

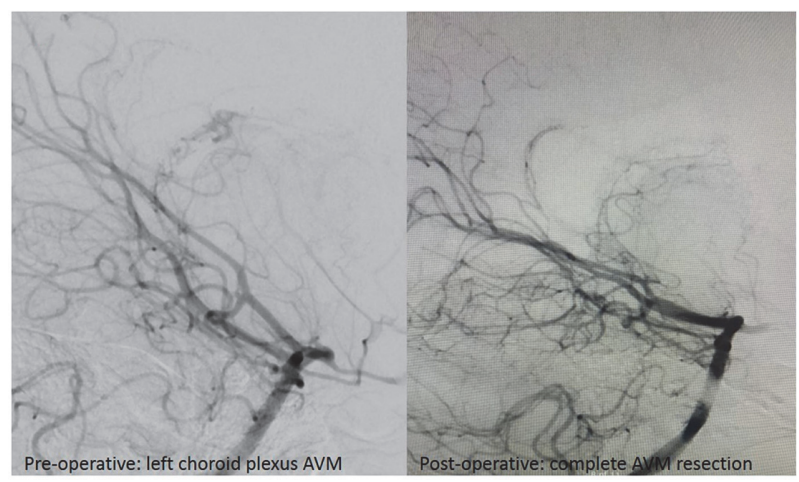

Abstract E-096 Figure 2

carcinoma metastasis with resultant IVH, and a lenticulostriate artery aneurysm with resultant basal ganglia hemorrhage. All patients had successful lesion resection, confirmed on postoperative imaging. There were no surgical complications.

Conclusions In this technical report, we demonstrate the effective use of a minimally invasive tubular retractor system for resection of various deep-seated vascular malformations and hemorrhagic masses. In all seven patients, lesions were successfully resected and no patient suffered surgical complications. This report shows the potential for minimally invasive techniques to adequately treat a wide variety of subcortical vascular lesions. This technique could revolutionize common approaches to open cerebrovascular surgery by mitigating neurological consequences of parenchymal insult relating to surgical retraction. Further research will determine whether minimally invasive techniques significantly improve patient outcomes.

Disclosures R. Achey: None. M. Bain: 2; C; Stryker, Cerenovus, Microvention, Rebound Therapeutics.

\section{E-097 SIMULTANEOUS TRANSARTERIAL AND TRANSVENOUS CONTRAST INJECTION TO REVEAL THE CONNECTION POINT FOR TREATMENT OF CAROTID CAVERNOUS FISTULA-TECHNICAL NOTE}

C Rupareliya* , J Fraser, L Sheikhi. University of Kentucky, Lexington, KY

10.1136/neurintsurg-2021-SNIS.192
Introduction Accessing cavernous sinus (CS) via inferior petrosal sinus (IPS) may become difficult sometimes for the treatment of carotid-cavernous fistulas (CCFs). We report a technique for simultaneous transarterial and transvenous contrast injection resulting in well opacification of IPS and fistulous point resulting in a successful entry using microcatheter.

Material and Methods A 58-year-old male was referred to our academic medical center for three weeks of right eye pain, now complicated by redness, diplopia, and blurry vision. Magnetic Resonance Imaging (MRI) brain at the outside hospital revealed a hemorrhagic lesion in right parietotemporal region. Computerized tomography-angiogram (CTA) of the head revealed filling of cavernous sinus during an arterial phase suspicious for CCF.

Under general anesthesia, after accessing right common femoral artery, 4F berenstein catheter was advanced over 0.035 angled glide wire to the proximal right internal carotid artery. Contrast injected through ICA showed CS but not the IPS [figure 1(a)]. Through the left common femoral vein, synchro 2 soft microwire was advanced through echelon 10 microcatheter. Venous guide catheter was advanced into right internal jugular vein (IJV) and distal access catheter was placed into sigmoid jugular junction. Injected contrast revealed IPS, but not CS [figure 1(b)]. A subsequent simultaneous hand injection with the microcatheter within the IPS and the diagnostic catheter in the left ICA elucidated the arteriovenous

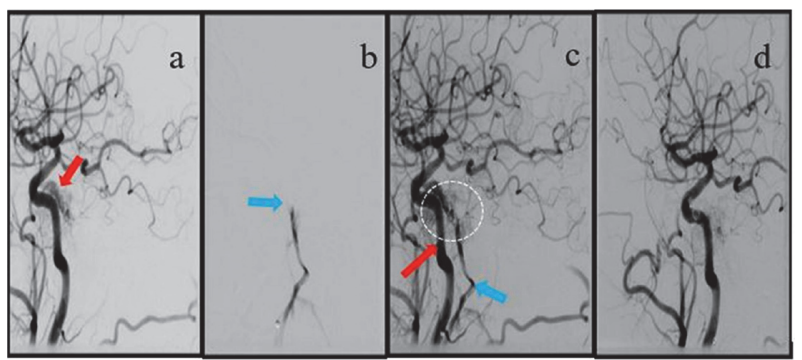

Abstract E-097 Figure 1 (a) There is flow into cavernous, but no visible flow through the IPS (b) Injection into right IJV opacifies half of IPS, but not the fistulous point or cavernous sinus (blue arrow) (c) Simultaneous injection into the right ICA (red arrow) and right IJV (blue arrow) pacifying the connection allowing subsequent navigation (white dashed circle) (d) Right iCA injection after successful coil embolization showing resolution

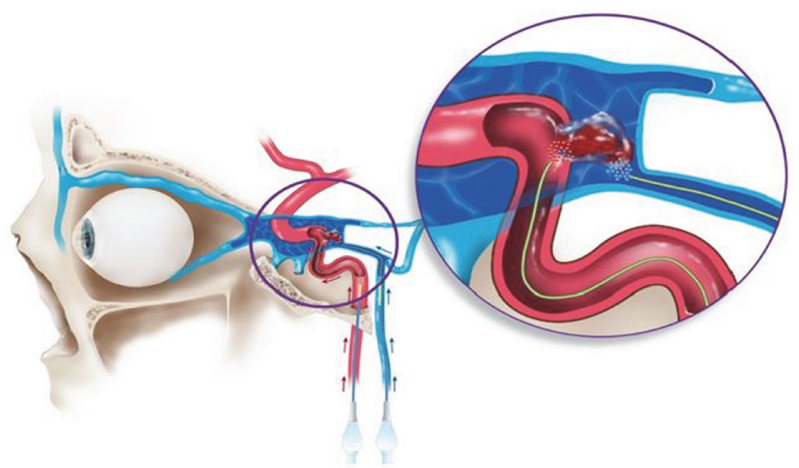

Abstract E-097 Figure 2 Illustration depicting simultaneous transarterial and transvenous contrast injection technique with point of connection magnified inside the circle 